\title{
Individual Cognitive Stimulation Therapy in Alzheimer's Disease: A Randomized Controlled Trial
}

\author{
Yu-chen Qiao \\ Xuanwu hospital, Capital Medical University \\ Hong Chang \\ Xuanwu hospital, Capital Medical University \\ Rui Wang \\ Xuanwu hospital, Capital Medical University \\ Jia-Mei Wang \\ Xuanwu hospital, Capital Medical University \\ Xiao-ying Wang \\ Xuanwu hospital, Capital Medical University \\ Yan Yu \\ Xuanwu hospital, Capital Medical University \\ Xin Yang \\ Xuanwu hospital, Capital Medical University \\ Jia Liu ( $\square$ Jason_liu1984@163.com ) \\ Xuanwu hospital, Capital Medical University
}

\section{Research Article}

Keywords: Cognition, Individual Cognitive Stimulation Therapy, Dementia, Alzheimer's disease

Posted Date: February 1st, 2021

DOl: https://doi.org/10.21203/rs.3.rs-138365/v1

License: (c) (i) This work is licensed under a Creative Commons Attribution 4.0 International License. Read Full License 


\section{Abstract}

Background: Individual cognitive stimulation therapy (ICST) can benefit cognition and quality of life for people with dementia, but the evidence for nurses-led individual cognitive stimulation interventions is limited.

Objectives: The current study aimed to develop the process of planning and implementing ICST, and evaluate the effect of ICST on cognitive function and therapeutic effect in patients with mild Alzheimer's disease (AD).

Methods: It was a randomized controlled trial lasting for a half year. 38 patients with mild AD were recruited. The control group was given the "Individual Cognitive Stimulation Therapy Manual for AD" to deliver the sessions at home. The intervention group was given 90 minutes 'cognitive stimulation sessions, completed up to three times weekly over 24 weeks. The outcomes were measured at baseline and 24weeks by the scales widely used in AD evaluation (MMSE, MoCA and ADL).

Results: There were statistical significance between the two groups, especially the scores of memory, delayed memory in MMSE and scores of memory, delayed memory, immediate memory, attention in MoCA were significantly improved in the intervention group compared with control group. ICST show positive effects that may help preserve memory in mild AD.

Conclusions: The ICST can improve the memory and attention cognitive domains in patients with mild AD. And the feedback from patient and caregiver in ICST was favorable.

\section{Introduction}

Dementia is featured by the impairment of cognitive functioning, leading to significant difficulty in daily life, learning, work, and social communication skills [1]. Alzheimer's disease (AD) is the most common phenotype, accounting for $50-70 \%$ of dementia [2]. Epidemiological data show that the prevalence of $A D$ in China is 3.21\% [3] for the people over 65 years old. That is, about 5 million AD patients live in China. The direct and indirect cost of treatment and care in total is about one trillion yuan one year [4]. In addition to lose memory, abnormal perception, emotion, and behavior are also frequent in $A D$, which aggravate the familiar and social burden [5]. Interventions for AD include pharmacological and non-pharmacological treatment. At present, no drugs can prevent or modify the disease progression. Among them, ICST is an important non-pharmacological method and thought to be a possible supplement to maintain the cognitive functioning in $A D$ with increased use in clinical practice.

CST refers to the use of non-specific cognitive interventions via team activities or discussions, such as handwork, topic discussion, and digital maze task, in order to achieve the effect of improving the overall cognitive function or social function of AD patients [6]. Patients with mild to moderate dementia engaging to group CST has been recommended by the National Institute for Health and Care excellence (NICE) [7]. In addition, a Cochrane study found [8] that benefits on cognitive function was evidently maintained during a follow-up of 1-3 months after CS intervention for people with mild to moderate dementia. Also, CST is cost-effective and comparable efficacy to anti-dementia drugs. Furthermore, ICST was developed to cater for those people with dementia who unable or unwilling to engage in groups owing to restricted resources, physical inconvenience, and personal preferences [9], Similarly, ICST is based on the principles of "use it or lose it", including mental stimulation, reminiscence and reality orientation may contribute to slow cognitive decline, evidence that activation of neurons may increase neuronal function and survival [10]. The present evidence suggests that delivery one to one is more effective than in a group [11]. Therefore, ICST is getting more and more attention from the international scholars. Indeed, ICST followed tailoring and personalization principles that increase the patients' participation in the CS, which yield positive effect on cognition function and social activity [12].

This suggest ICST led by family caregiver may produce benefits for both the person and caregiver. However, there have some adherence difficulty conducting all session as planned led by family caregivers who suggested that extra support to help to improve compliance [13]. Therefore, further develop a professional nurse-led of ICST would be increasing the attendance of the intervention for people unable to complete all session. Promoting people with dementia to complete the session needs professional nurse in a creative and enjoyable way according patients' preferences whenever possible trouble happens, but fewer investigate on the concrete process of planning or implementing the intervention main-conducted by nurse.

Page 2/14 
In this paper, we aimed to put forward a personalized cognitive stimulation scheme for patients with mild AD, which was strict quality controlled and ensured the attendance by nurse-led in the process of implementing and evaluating the effects of intervention. So that, we can determine the therapeutic effect of ICST, a non-drug therapy, on the cognitive function of AD patients.

\section{Methods}

\section{Study design and participant}

Randomized controlled trial was conducted(Figure.1). A total of 38 patients with mild AD from memory clinic, Xuanwu hospital, Capital Medical University from March to September 2018 were enrolled as following criteria. Participants were randomly assigned into either intervention group $(n=19)$ or control group $(n=19)$. Participant met the Inclusion criteria: $\otimes$ clinically diagnosed as AD; $\varangle$ mild dementia (Clinical Dementia Rating, CDR 1 point); $\varangle$ with formal caregivers. Formal caregivers must be available for at least 4 days per week and over 4 hours per day for patients, and must be able to accompany patients for each visit; $\nabla$ able to express informed consent. $\nabla$ had some ability to communicate and understand communication. $\llbracket$ see and hear well enough to engage in activities. $\otimes$ had no major physical illness or disability affecting participation $\otimes$ had prescribed to Anticholinesterase inhibitors for more than 3 months. Exclusion criteria: $\otimes$ Dementia caused by other brain diseases, such as central nervous system demyelinating diseases, tumors, hydrocephalus, and central system infections (syphilis, AIDS, Creutzfeldt-

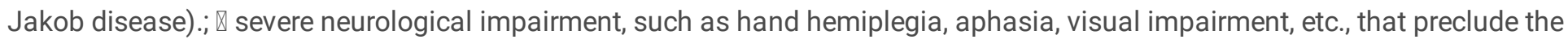

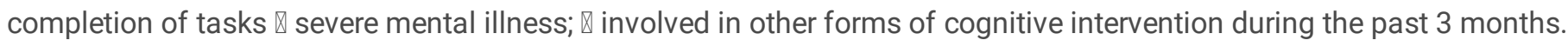

\section{Ethics statement}

This study was obtained ethical approval from the Ethics Committee of Xuanwu Hospital, Capital Medical University (2015010). This trail was registered as a ClinicalTrials.gov (NCT02640716冈the first registration on 29/12/2015)

\section{Randomization and blinding}

After consent and baseline assessment, the randomization is generated by a researcher who is not involved in this study, and these participants were divided into control and intervention groups according to a random numbers table. According to the order of admission of the research subjects, the research subjects are numbered in advance. After participants were randomized, baseline and finally assessment data were collected by blinded researchers. And, Statisticians who are blind to the allocation performed the finally analysis.

\section{Interventions}

In this study, ICST is designed as a dynamic, continuous process, including the following three steps: preparation before intervention, developing a plan and implementation.

The members of the research team are composed of neurologists, nurses and neuropsychologists. Neurologists are responsible for the clinical diagnosis. Nurses who were professional trained by occupational therapist following ICST manual [14], are in charge of the development and implementation of ICST programs. Neuropsychologists are responsible for the neuropsychological evaluation of each participant at baseline and after intervention. Neurologists, neuropsychologists and data analysts were blinded to the group assignment.

Writing the manual on CST for AD, containing 6 parts: What is AD, Performance of AD, Pharmacological and NonPharmacological Treatment of AD, Home Care Approaches and Skills, Significance and Principals of CST, and Process and methods of CST. A detailed overview of the manual is presented in Table 1. The manual was sent to the participants and their family caregivers. And the participants in intervention group were trained by the nurses.

Neurologists made the clinical diagnosis according to neuroimaging, laboratory examination, and clinical examination. Neuropsychologists provided a comprehensive and detailed assessment of the cognitive function and daily activities. 
Nurses and family caregivers were trained by an unblinded professional researcher before the intervention. The first part of the training session introduces the context of CST Manual for AD, and explains the themes, session structure and key principles of CST. And standard toolkits were sent to the patients and their caregivers, which materials were related to this whole themes of activities, be used for each session according to this CST schemes.

Given two nurses were established CST files for AD patients in the nursing clinic, including: patient characteristic data, assessment outcomes of cognitive function and ability of daily life. Besides, the problems and difficulties experienced by the patients during this training were recorded after each training.

\section{Intervention group}

Patients participating in ICST was performed by nurses at Cognitive Functional Training Nursing Outpatient of Xuanwu hospital, where the nurses developed an ICST program for each participant considering cognitive abilities, hobbies and interests based on their diagnosis and cognitive function assessment. Intervention programs include individualized training and group training. Individualized training was the handwork, such as doll making, collage making and creative production. After the first intervention, the difficulty was adjusted according to the case of patient completing the program, and the individualized intervention plan was revised and finalized. Group training takes the form of team activities such as finger exercises and tossing objects.

The frequency of cognitive stimulation was 3 times per week, each session was 90 minutes, lasting for a total of 24 weeks. Each patient underwent individualized training for 66 times and group training for 6 times. Individualized training session included: 10 minutes communication between patient and nurse/caregiver on the following topics: hobbies, likes, dislikes, everyday routine, everyday limitations, previous profession/occupation, family dynamics and life history, observation of emotional responses, and explanation of training content to patient; 60 minutes: according to each patients' main concerns and preferred hobbies, nurse formulated two tailored activities for each patient, and nurse would require patient to recall the training steps to strengthen memory, and fully use as many sensory stimuli as possible during making handwork. Specially, nurse mostly focus on patients' opinions rather than facts, and provide opportunities to stimulate language and closely bound the relationship; 10-minute communication about the details of training, e.g. difficulty encountered during the training by patients and caregivers; 10 -minute explanation of disease knowledge, including the clinical symptoms, drug- and non-drug therapy, nursing principles, complication care, behavior treatment. Group training occurred on the $4^{\text {th }}$ week, $8^{\text {th }}$ week, $12^{\text {th }}$ week, $16^{\text {th }}$ week, $20^{\text {th }}$ week, and $24^{\text {th }}$ weeks. There were 5-6 people per group, with 60-minute team activities 30-minute topical discussion about the prevention of dementia, healthy lifestyle for elderly, home self-care and aerobic exercise. This group events are designed to promote patient's participation and motivation in a happy, respectful and decent way, and build exchange among people with dementia in a social setting.

\section{Control group}

The participants and their families in the control group were self-trained at home according to the manual and a standard tookit. The caregiver records the problems and difficulties during the training, as well as the number of completed sessions, and nurses carried out 10 minutes of health education and solved questions about cognitive stimulation approach though mobile phone 3 times per week.

\section{Data collection}

All outcome measures were completed at baseline, 24 weeks after baseline by trained psychologists who were blinded to allocation information.

\section{Quality control and assurance}

A scheme of ICST was designed and implemented by the same nurse according to the protocol. The number of attendance and date of completing were recorded by the nurse for each person on a note, whereby in each theme and the name of handwork. 
The total score of MMSE [15] 25-30 represented normal cognitive function, 21-24 represented mild cognitive impairment, 14-20 represented moderate cognitive impairment, 13 and below represented severe cognitive impairment. The criteria were adjusted for education. Chinese version of MMSE was with good reliability. Internal consistency Cronbach 's a coefficient is 0.833 and the reliability of the retest is 0.924 .

The total score of MoCA [16] was 30. The score was adjusted for education. A score of 26 or above was normal. The sensitivity of Chinese version MOCA was 0.92 , the specificity was 0.84 , the reliability coefficient of retest was 0.86 , while the internal consistency Cronbach's a coefficient was 0.82 .

A full score of Barthel [17] 100 indicated full independence for activities of daily life (ADL). Less than 20 indicated very heavy dependence, 25-45 indicated heavy dependence, 50-70 indicated moderate dependence, 75-95 indicated mild dependence. Its retest reliability was 0.89 .

\section{Statistical Analysis}

This study made use of SPSS25.0 software for statistical analysis. The measurement data were expressed as mean \pm standard deviation. The two groups were compared using independent sample t tests, and count data were analyzed by $\mathrm{X}^{2}$ tests. The categorical variables of demographic and clinical variables that were related to the outcome scores were analyzed by one-way analysis of variance (ANOVA) and continuous variables were analyzed by linear regression. The significance threshold was set at $\mathrm{a}=0.05$.

\section{Results}

A total of 38 participants met the inclusion and exclusion criteria and were recruited. Six participants withdrew from the study during this research period. On the one hand, owing to one with unwilling to continue and two with changing residence, the number of intervention group was 16 . One the other hand, the reason of new onset of medical problems with two and changing residence with one, three participants can't engage in the final assessment, and the number of control group was 16.

Table 2 shows baseline characteristics of participants. There was no significant difference between the two groups in age, sex and education. Table 3 shows the efficacy measures at the endpoint before of follow-up. There was no significant difference in the total score of MMSE and MoCA, as well as the scores of each cognitive domain between the two groups. Table 4 shows the efficacy based on the changes of ADL in two groups with no significant difference in total score. Table 5 presents the efficacy outcome scores after intervention. Memory, delayed memory scores in MMSE were significantly different between the two groups. Memory, delayed memory, immediate memory, attention scores in MoCA were significantly different between the two groups $(P<0.05)$. MMSE and MoCA scores from pre-treatment to post-treatment were independent of to any baseline demographic or clinical variables in Table6. The number of sessions completed by patients with $A D$ and their family caregivers ranged from 0 to 72 over the 24 weeks in Figure2.

\section{Discussion}

The interventions applied during a 6-month period did not improve the cognitive function of mild AD patients. Through 6-month cognitive stimulation, the total MMSE score was not significantly different between the groups. The finding was consistent with the results of Kim and Suzuki $[18,19]$. Although experts have not yet reached a consensus on the frequency, and intensity of cognitive stimulation, the improvement or maintenance of cognitive function in AD patients should be long-term for patients and their families. Cognitive stimulation is unlikely to produce a significant effect in a short-term. Therefore, cognitive stimulation may play a therapeutic effect with persistent use. After 6-month intervention, the MoCA scores were not significantly different between the groups, which was consistent with the results by Polito [20]. The reason could be that MoCA is more comprehensive with more complicated content, which requires higher level of cognition to complete. Concerning the process of cognitive stimulation, close cooperation between caregivers and nurses for enough intervention periods is required with 
sufficient confidence and patience in implementing cognitive stimulation for patients. Moreover, a multi-modal cognitive stimulation model [21] may play a role in improving the overall cognitive function of AD patients, such as the improving and optimizing stimulation methods, combining lifestyle interventions with original methods, increasing aerobic exercise, implementing memory therapy, implementing Tai Chi and yoga programs, and combining virtual reality with neural control techniques such as transcranial magnetic stimulation.

Cognitive stimulation is proven to have a positive effect on certain cognitive aspects in AD patients. After 6-month intervention, the scores of memory, delayed memory in MMSE and memory, delayed memory, immediate memory, attention in MoCA was significantly improved in the treatment group. Longitudinal analysis showed a significant decrease in the control group in orientation, calculation abilities, and language function based on MMSE scores, with only memory and visual space function maintained. All cognitive domains were significantly decreased in the control group in MoCA. Hall et al, pointed out that cognitive stimulation can improve the overall cognitive function of people with dementia, especially in terms of language ability, memory, and orientation, which is consistent with our results [22]. Cognitive stimulation mainly emphasizes language communication, and helps people with dementia to preserve grammatical function, and encourage patients to actively think, ask questions, and express their views through strengthening the neural pathways of language. It is worth noting that the current duration of cognitive stimulation varies from 1 to 3 months, so that the results need to be further verified. Cognitive stimulation also had a significant effect on memory [23], which may be helpful to AD's earliest memory impairment. Hall et al. noted that cognitive stimulation could directly strengthen learning and memory via memory encoding and extraction capabilities, and create positive and optimal learning environment for patients.

In this study, there was no statistical difference in ADL scores between the two groups. This result is consistent with the results of Kurz [24] and others. Indeed, cognitive stimulation mainly focuses on the cognitive function, not so much on the patient's daily life. Cognitive stimulation may not have the effect in improving the daily living ability. However, cognitive stimulation can improve the memory and attention ability of AD patients, which may indirectly promote the maintenance of daily living abilities. In Bergamaschi's study, ADL was significantly improved $(p<0.05)$, suggesting that prolonged intervention period of cognitive stimulation may improve the daily activity ability in AD patients[25]. 10-week intervention in Giuli's study at 45 minutes each, 10 times a week, showed a significant increase in ADL $(p<0.05)$ and L-ADL $(p<0.001)$, suggesting that high-frequency cognitive stimulation interventions may play a positive role in affecting daily life ability [26]. However, it is necessary to evaluate the physical condition and mood of AD patients in detail, to avoid intensive cognitive stimulation discomforting the patients, which may affect the effect of intervention.

\section{Limitations}

The intervention period is short and sample size is small because it was conducted with patients applying to a health institution. the effects of cognitive stimulation on cognitive function and life self-care ability need to be further discussed. In addition, although the specific cognitive tasks is lacking, the present results may ultimately contribute to the generalization.

\section{Future research}

The current research results suggest several ways for future research, and the potential value of conducting a larger-scale RCT. Researchers will monitor patients through longer cognitive interventions, comparing the intervention effects of intervention for 12 months, 18 months and 24 months, to evaluate the longer term impact.

\section{Conclusions}

A nurse-led ICST is feasible to perform with people having mild AD, and the main findings suggest that it has positive effects on memory and attention cognitive function. Its potential to serve as a prevent cognitive decline intervention starting at earliest possible time in the developing regions where dementia epidemic.

\section{Declarations}




\section{Funding}

Not applicable.

\section{Protocol}

The protocol was provided on http://ClinicalTrials.gov/NCT02640716

Acknowledgments

We appreciate the cooperation of the staff of the Guangdong Provincial Hospital of Chinese Medicine.

\section{Authors' contributions}

YCQ, HC and JL conceived and designed the study. RW, JMW and XYW recruited the participants, collected the data for the manuscript and provided substantial feedback. YY, XW, XY and JL analyzed and interpreted the data. YCQ, HC and JL wrote the first draft of the manuscript. All authors read and approved the final manuscript.

\section{Availability of data and materials}

The datasets used and/or analyzed during the current study are available from the corresponding author on reasonable request.

\section{Ethics approval and consent to participate}

This study was on accordance with relevant guidelines and regulations (Declaration of Helsinki) and approved by the Ethics Committee of Xuanwu Hospital, Capital Medical University (2015010). All the participants provided written informed consent. For the certain participants recognized as having cognitive impairment and/or severe illness, we obtained proxy consent from a family member or another supportive adult on their behalf.

\section{Consent for publication}

Not applicable.

\section{Competing interests}

The authors declare that they have no competing interests.

\section{References}

1. Michael B. First, MD. Diagnostic and statistical manual of mental disorders, 5th edition, and clinical utility. J Nerv Ment Dis, 2013;201(9):727-729.

2. Hugo J, Ganguli M. Dementia and cognitive impairment: epidemiology, diagnosis, and treatment. Clin Geriatr Med. 2014;30(3):421-442.

3. Jia J, Wang F, Wei C, Zhou A, Jia X, Li F, et al. The prevalence of dementia in urban and rural areas of China. Alzheimers Dement. 2014;10(1):1-9.

4. Jia J, Wei C, Chen S, Li F, Tang Y, Qin W, et al. The cost of Alzheimer's disease in China and re-estimation of costs worldwide. Alzheimers Dement. 2018;14(4):483-491.

5. Qiao Yuchen, Wang Jiamei, Yu Yan, Sun Jing, Zhang Yue, Chang Hong. Ward-outpatient-family integrated management model construction among patients with Mild Cognitive Impairment. Chinese Nursing Management, 2017; v.17(12):78-81.

6. Rai H, Yates L, Orrell M. Cognitive Stimulation Therapy for Dementia. Clin Geriatr Med. 2018;34(4):653-665.

7. National Institute for Health and Care Excellence (UK). Dementia: Assessment, management and support for people living with dementia and their carers. London: National Institute for Health and Care Excellence (UK); 2018. 
8. Woods B, Aguirre E, Spector AE, Orrell M. Cognitive stimulation to improve cognitive functioning in people with dementia. Cochrane Database Syst Rev. 2012;15(2):CD005562.

9. Yates LA, Leung P, Orgeta V, Spector A, Orrell M. The development of individual cognitive stimulation therapy (iCST) for dementia. Clin Interv Aging. 2014;30(10):95-104.

10. Salthouse TA. Mental Exercise and Mental Aging: Evaluating the Validity of the "Use It or Lose It" Hypothesis. Perspect Psychol Sci. 2006;1(1):68-87.

11. Van't Leven N, Prick AE, Groenewoud JG, Roelofs PD, de Lange J, Pot AM. Dyadic interventions for community-dwelling people with dementia and their family caregivers: a systematic review. Int Psychogeriatr. 2013;25(10):1581-603.

12. Rita Maldonado Branco, Joana Quental \& Óscar Ribeiro. Personalised participation: an approach to involve people with dementia and their families in a participatory design project. Codesign, 2017;13(2):127-143.

13. Leung P, Yates L, Orgeta V, Hamidi F, Orrell M. The experiences of people with dementia and their carers participating in individual cognitive stimulation therapy. Int J Geriatr Psychiatry. 2017;32(12):e34-e42.

14. Katzman R, Zhang MY, Ouang-Ya-Qu, Wang ZY, Liu WT, Yu E, et al. A Chinese version of the Mini-Mental State Examination; impact of illiteracy in a Shanghai dementia survey. J Clin Epidemiol. 1988;41(10):971-978.

15. Orgeta V, Leung P, Yates L, Kang S, Hoare Z, Henderson C, et al. Individual cognitive stimulation therapy for dementia: a clinical effectiveness and cost-effectiveness pragmatic, multicentre, randomised controlled trial. Health Technol Assess. 2015;19(64):101-108.

16. Nasreddine ZS, Phillips NA, Bédirian V, Charbonneau S, Whitehead V, Collin I, et al. The Montreal Cognitive Assessment, MoCA: a brief screening tool for mild cognitive impairment. J Am Geriatr Soc. 2005;53(4):695-699.

17. Teasell R. Stroke recovery and rehabilitation. Stroke. 2003;34(2):365-366.

18. Kim HJ, Yang Y, Oh JG, Oh S, Choi H, Kim KH, et al. Effectiveness of a community-based multidomain cognitive intervention program in patients with Alzheimer's disease. Geriatr Gerontol Int. 2016;16(2):191-199.

19. Suzuki H, Kuraoka M, Yasunaga M, Nonaka K, Sakurai R, Takeuchi R, et al. Cognitive intervention through a training program for picture book reading in community-dwelling older adults: a randomized controlled trial. BMC Geriatr. 2014;21(14):122.

20. Polito L, Abbondanza S, Vaccaro R, Valle E, Davin A, Degrate A, et al. Cognitive stimulation in cognitively impaired individuals and cognitively healthy individuals with a family history of dementia: short-term results from the "Allena-Mente" randomized controlled trial. Int J Geriatr Psychiatry. 2015 ;30(6):631-638.

21. Maresova P, Tomsone S, Lameski P, Madureira J, Mendes A, Zdravevski E, et al. Technological Solutions for Older People with Alzheimer's Disease: Review. Curr Alzheimer Res. 2018;15(10):975-983.

22. Hall L, Orrell M, Stott J, Spector A. Cognitive stimulation therapy (CST): neuropsychological mechanisms of change. Int Psychogeriatr. 2013;25(3):479-89.

23. Nicoll JA, Wilkinson D, Holmes C, Steart P, Markham H, Weller RO. Neuropathology of human Alzheimer disease after immunization with amyloid-beta peptide: a case report. Nat Med. 2003;9(4):448-452.

24. Kurz A, Thöne-Otto A, Cramer B, Egert S, Frölich L, Gertz HJ, et al. CORDIAL: cognitive rehabilitation and cognitive-behavioral treatment for early dementia in Alzheimer disease: a multicenter, randomized, controlled trial. Alzheimer Dis Assoc Disord. 2012;26(3):246-253.

25. Bergamaschi S, Arcara G, Calza A, Villani D, Orgeta V, Mondini S. One-year repeated cycles of cognitive training (CT) for Alzheimer's disease. Aging Clin Exp Res. 2013;25(4):421-426.

26. Giuli C, Papa R, Lattanzio F, Postacchini D. The Effects of Cognitive Training for Elderly: Results from My Mind Project. Rejuvenation Res. 2016;19(6):485-494.

\section{Tables}

\section{Table 1 Overview of CST Manual for AD}


1. What's Why is people aging?

Alzheimer's

When will the brain age?

What's cognitive impairment?

What's AD?

2.

Performance of

$A D$
Common manifestations of $A D$

Early symptoms of $A D$

How to distinguish normal aging from $A D$ ?

Will memory loss occur only in the elderly?

Why do many older people become sensitive and vulnerable?
3 .

Pharmacological and Non-

Pharmacological

Treatment of $A D$
Precautions for Pharmacological Therapy

Non-pharmacological treatment methods
4. Home Care Approaches and Skills Significance and Principals of ICST

\section{Diet management}

Emotional management

Overcoming sleep disorders

Home security

Reasonable arrangement of activities

Communicating with the elderly people with $A D$

\section{What's ICST?}

The importance of ICST

The Principals of ICST
6. Processes and methods of ICST

\section{Frequency and total sessions:}

72 sessions delivered by the nurse/family caregiver for 90 minutes, three times a week, over 24 weeks.

Procedure:

Using a standardised toolkits according to the manual and key principles of ICST.

Methods:

10 minutes communication between patient and nurse/caregiver on the following topics: hobbies, likes, dislikes, everyday routine, everyday limitations, previous profession/occupation, family dynamics and life history, number games, childhood, orientation, associated words,slogan, famous faces, be creative, categorizing object, physical games, food. 
Table 2 Baseline characteristics

\begin{tabular}{|c|c|c|c|}
\hline & $\begin{array}{l}\text { Intervention group } \\
(\mathrm{n}=16)\end{array}$ & Control group $₫ n=16 \rrbracket$ & $P$ \\
\hline Age $\ y \rrbracket$ & $62.69 \pm 8.97$ & $66.56 \pm 11.68$ & 0.107 \\
\hline Diagnosis of $A D(y)$ & $2.51 \pm 1.01$ & $1.94 \pm 0.99$ & 0.117 \\
\hline Comorbidities & $1.61 \pm 0.67$ & $1.21 \pm 0.66$ & 0.105 \\
\hline \multicolumn{4}{|l|}{ Sex『\%凹 } \\
\hline male & $9 \rrbracket 56.25 \rrbracket$ & $6 \rrbracket 37.50 \rrbracket$ & \multirow[t]{2}{*}{0.288} \\
\hline female & $7 \rrbracket 43.75 \rrbracket$ & $10 \rrbracket 62.50 \rrbracket$ & \\
\hline \multicolumn{4}{|l|}{ Marital status } \\
\hline married\% & $15 \llbracket 93.75 \rrbracket$ & 16ه100.00》 & \multirow[t]{2}{*}{0.310} \\
\hline Unmarried, divorced or widowed \% & $1 \rrbracket 6.25 \rrbracket$ & $0 \rrbracket 0.00 \rrbracket$ & \\
\hline \multicolumn{4}{|l|}{ Living with $₫ \% \rrbracket$} \\
\hline Alone & $1 \rrbracket 6.25 \rrbracket$ & $1 \rrbracket 6.25 \rrbracket$ & \multirow[t]{2}{*}{0.659} \\
\hline Family members & $13 \llbracket 81.25 \rrbracket$ & $11 \rrbracket 68.75 \rrbracket$ & \\
\hline others & $2 ه 12.5 \bigotimes$ & $4 \rrbracket 25.00 \rrbracket$ & \\
\hline \multicolumn{4}{|l|}{ Education $₫ \% \rrbracket$} \\
\hline primary school & $1 \rrbracket 6.25 \rrbracket$ & $1 \rrbracket 6.25 \rrbracket$ & \multirow[t]{4}{*}{0.477} \\
\hline Junior high school & $6 \rrbracket 37.50 \rrbracket$ & $6 \rrbracket 37.50 \rrbracket$ & \\
\hline High school & $4 \rrbracket 25.00 \rrbracket$ & 1ه6.25区 & \\
\hline College/university & $5 \rrbracket 31.25 \rrbracket$ & $8 \rrbracket 50.00 \rrbracket$ & \\
\hline
\end{tabular}

Table 3 Results of baseline assessments in the two groups 


\begin{tabular}{|c|c|c|c|c|}
\hline Scales & $\begin{array}{l}\text { Intervention group } \\
\bigotimes n=16 \rrbracket\end{array}$ & $\begin{array}{l}\text { Control group } \\
\bigotimes n=16 \rrbracket\end{array}$ & $\mathrm{t}$ & $P$ \\
\hline MMSE & $21.06 \pm 3.678$ & $20.38 \pm 4.193$ & 0.493 & 0.626 \\
\hline Orientation & $6.31 \pm 2.024$ & $5.13 \pm 2.247$ & 1.571 & 0.127 \\
\hline Calculate & $3.25 \pm 1.183$ & $2.88 \pm 1.204$ & 0.889 & 0.381 \\
\hline Visual space & $0.44 \pm 0.512$ & $0.63 \pm 0.500$ & -1.048 & 0.303 \\
\hline Memory & $4.44 \pm 1.209$ & $4.81 \pm 0.750$ & -1.054 & 0.300 \\
\hline Language & $6.56 \pm 0.964$ & $7.19 \pm 0.834$ & -1.961 & 0.059 \\
\hline immediate memory & $2.75 \pm 0.447$ & $2.94 \pm 0.250$ & -1.464 & 0.154 \\
\hline delayed memory & $1.69 \pm 1.014$ & $1.88 \pm 0.719$ & -0.603 & 0.551 \\
\hline MoCA & $15.19 \pm 4.102$ & $15.06 \pm 3.974$ & 0.088 & 0.931 \\
\hline Execution & $1.69 \pm 0.946$ & $1.88 \pm 0.806$ & -0.603 & 0.551 \\
\hline Visual space & $0.38 \pm 0.500$ & $0.69 \pm 0.479$ & -1.806 & 0.081 \\
\hline Attention & $2.50 \pm 0.632$ & $2.88 \pm 0.619$ & -1.695 & 0.100 \\
\hline Calculate & $2.38 \pm 1.258$ & $2.38 \pm 1.258$ & 0.000 & 1.000 \\
\hline Orientation & $3.19 \pm 1.471$ & $2.44 \pm 1.263$ & 1.547 & 0.132 \\
\hline Language & $4.13 \pm 0.806$ & $4.13 \pm 0.885$ & 0.000 & 1.000 \\
\hline Memory & $6.06 \pm 2.144$ & $6.50 \pm 1.897$ & -0.611 & 0.546 \\
\hline immediate memory & $5.94 \pm 1.948$ & $6.18 \pm 1.470$ & -0.410 & 0.685 \\
\hline delayed memory & $0.13 \pm 0.500$ & $0.31 \pm 0.602$ & -0.958 & 0.346 \\
\hline
\end{tabular}

Table 4 Efficacy measures ADL comparison before-after in two groups

\begin{tabular}{|c|c|c|c|c|c|c|c|c|}
\hline \multirow[t]{3}{*}{ Characteristics } & Interventic & group & \multirow[t]{3}{*}{$x^{2}$} & \multirow[t]{3}{*}{$P$} & \multirow{2}{*}{\multicolumn{2}{|c|}{ Control group $\varangle n=16 \rrbracket$}} & \multirow[t]{3}{*}{$x^{2}$} & \multirow[t]{3}{*}{$P$} \\
\hline & \multicolumn{2}{|l|}{$(n=16)$} & & & & & & \\
\hline & Before & After & & & Before & After & & \\
\hline normal & $13 \llbracket 81.3 \rrbracket$ & $15 \llbracket 93.8 \rrbracket$ & 1.143 & 0.285 & 13 & 14 & 0.237 & 0.626 \\
\hline Abnormal & $3 \otimes 18.8 \rrbracket$ & $1 \otimes 6.3 \rrbracket$ & & & 3 & 2 & & \\
\hline
\end{tabular}

Table 5 Results of assessments in the two groups during follow-up 


\begin{tabular}{|c|c|c|c|c|c|c|c|c|}
\hline \multirow[t]{2}{*}{ Scales } & \multicolumn{2}{|c|}{ Intervention group } & \multirow{2}{*}{$\begin{array}{l}\text { Difference } \\
\text { value }\end{array}$} & \multicolumn{2}{|l|}{ Control group } & \multirow{2}{*}{$\begin{array}{l}\text { Difference } \\
\text { value }\end{array}$} & \multirow[t]{2}{*}{$t$} & \multirow[t]{2}{*}{$P$} \\
\hline & Before & After & & Before & After & & & \\
\hline MMSE & $21.06 \pm 3.678$ & $19.69 \pm 5.618$ & $1.37 \pm 5.071$ & $20.38 \pm 4.193$ & $16.69 \pm 5.263$ & $3.68 \pm 4.83$ & -1.321 & 0.196 \\
\hline Orientation & $6.31 \pm 2.024$ & $5.75 \pm 2.864$ & $0.56 \pm 1.97$ & $5.13 \pm 2.247$ & $4.25 \pm 2.145$ & $0.88 \pm 1.543$ & -0.500 & 0.621 \\
\hline Calculate & $3.25 \pm 1.183$ & $3.00 \pm 1.461$ & $0.25 \pm 1.183$ & $2.88 \pm 1.204$ & $2.31 \pm 1.401$ & $0.56 \pm 0.209$ & -0.739 & 0.466 \\
\hline $\begin{array}{l}\text { Visual } \\
\text { space }\end{array}$ & $0.44 \pm 0.512$ & $0.31 \pm 0.479$ & $0.13 \pm 0.342$ & $0.63 \pm 0.500$ & $0.56 \pm 0.512$ & $0.06 \pm 0.574$ & 0.374 & 0.711 \\
\hline Memory & $4.44 \pm 1.209$ & $4.13 \pm 1.310$ & $0.31 \pm 1.537$ & $4.81 \pm 0.750$ & $3.38 \pm 1.258$ & $1.44 \pm 1.413$ & -2.156 & 0.039 \\
\hline Language & $6.56 \pm 0.964$ & $6.63 \pm 1.088$ & $-0.63 \pm 1.340$ & $7.19 \pm 0.834$ & $6.50 \pm 1.317$ & $0.68 \pm 1.195$ & -1.671 & 0.105 \\
\hline $\begin{array}{l}\text { immediate } \\
\text { memory }\end{array}$ & $2.75 \pm 0.447$ & $2.81 \pm 0.403$ & $-0.06 \pm 0.680$ & $2.94 \pm 0.250$ & $2.69 \pm 0.602$ & $0.25 \pm 0.447$ & -1.536 & 0.135 \\
\hline $\begin{array}{l}\text { delayed } \\
\text { memory }\end{array}$ & $1.69 \pm 1.024$ & $1.31 \pm 1.138$ & $0.38 \pm 1.147$ & $1.88 \pm 0.719$ & $0.56 \pm 0.814$ & $1.31 \pm 1.078$ & -2.382 & 0.024 \\
\hline MoCA & $15.19 \pm 4.102$ & $13.94 \pm 5.260$ & $1.25 \pm 4.796$ & $15.06 \pm 3.974$ & $11.56 \pm 4.131$ & $3.50 \pm 5.046$ & -1.293 & 0.206 \\
\hline Execution & $1.69 \pm 0.946$ & $1.44 \pm 0.964$ & $0.25 \pm 0.577$ & $1.88 \pm 0.806$ & $1.44 \pm 0.814$ & $0.43 \pm 1.315$ & -0.522 & 0.605 \\
\hline $\begin{array}{l}\text { Visual } \\
\text { space }\end{array}$ & $0.38 \pm 0.500$ & $0.31 \pm 0.479$ & $0.06 \pm 0.443$ & $0.69 \pm 0.479$ & $0.31 \pm 0.479$ & $0.38 \pm 0.500$ & -1.872 & 0.071 \\
\hline Attention & $2.50 \pm 0.632$ & $2.63 \pm 0.619$ & $-0.13 \pm 0.619$ & $2.88 \pm 0.619$ & $1.94 \pm 0.998$ & $0.94 \pm 0.997$ & -3.619 & 0.001 \\
\hline Calculate & $2.38 \pm 1.258$ & $2.13 \pm 1.408$ & $0.25 \pm 1.125$ & $2.38 \pm 1.258$ & $1.94 \pm 1.237$ & $0.43 \pm 1.093$ & -0.478 & 0.636 \\
\hline Orientation & $3.19 \pm 1.471$ & $2.75 \pm 1.770$ & $0.43 \pm 1.711$ & $2.44 \pm 1.263$ & $2.13 \pm 1.204$ & $0.31 \pm 0.873$ & 0.260 & 0.796 \\
\hline Language & $4.13 \pm 0.806$ & $3.94 \pm 0.998$ & $0.18 \pm 1.047$ & $4.13 \pm 0.885$ & $3.38 \pm 1.088$ & $0.75 \pm 1.125$ & -1.464 & 0.145 \\
\hline Memory & $6.06 \pm 2.144$ & $6.19 \pm 2.903$ & $-0.13 \pm 3.159$ & $6.50 \pm 1.897$ & $4.13 \pm 1.455$ & $2.37 \pm 1.928$ & -2.702 & 0.012 \\
\hline $\begin{array}{l}\text { immediate } \\
\text { memory }\end{array}$ & $5.94 \pm 1.948$ & $0.13 \pm 0.500$ & $-0.50 \pm 1.789$ & $6.19 \pm 1.470$ & $0.31 \pm 0.602$ & $2.06 \pm 1.731$ & -4.118 & 0.000 \\
\hline $\begin{array}{l}\text { delayed } \\
\text { memory }\end{array}$ & $6.43 \pm 2.159$ & $0.31 \pm 0.602$ & $-1.19 \pm 0.403$ & $4.13 \pm 1.455$ & $0.00 \pm 0.000$ & $0.31 \pm 0.602$ & -2.760 & 0.010 \\
\hline
\end{tabular}

Table 6 Correlation analysis between the baseline outcome and change of post intervention with demographic variables

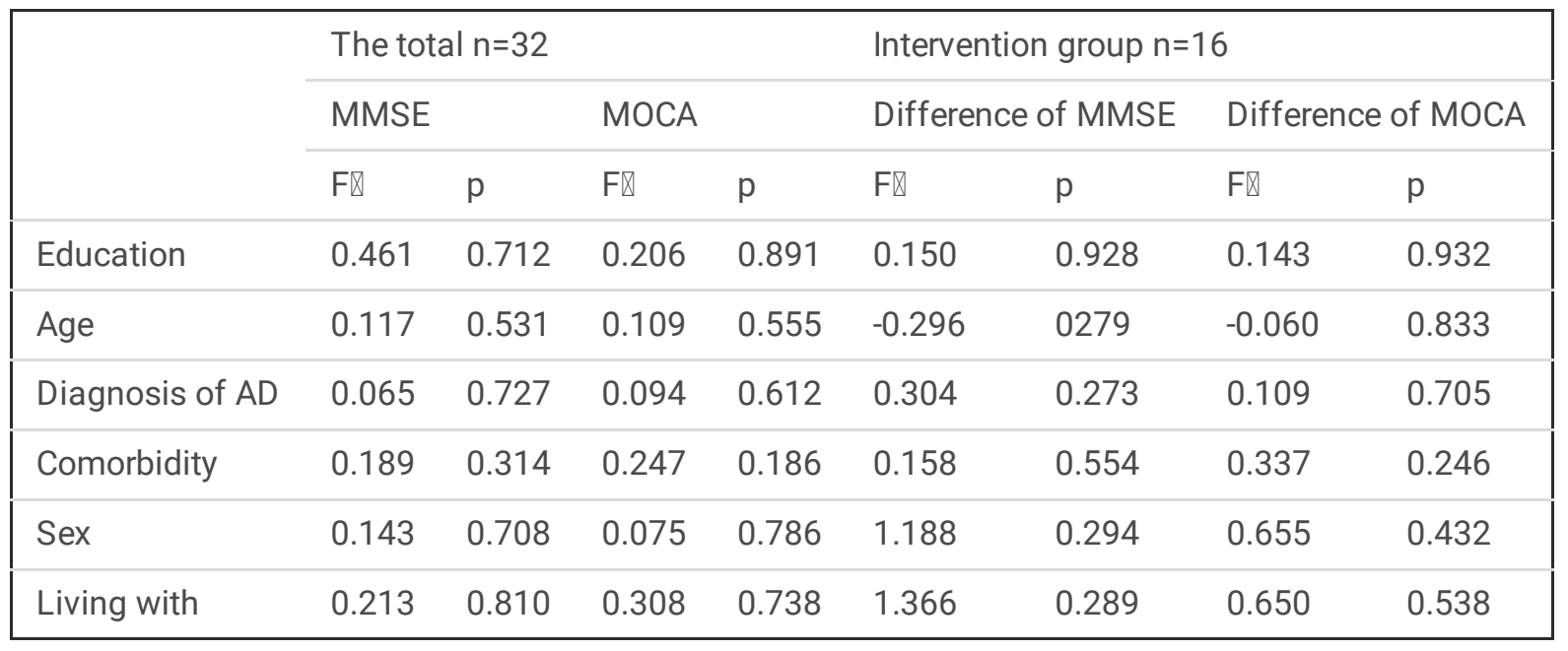


Figures

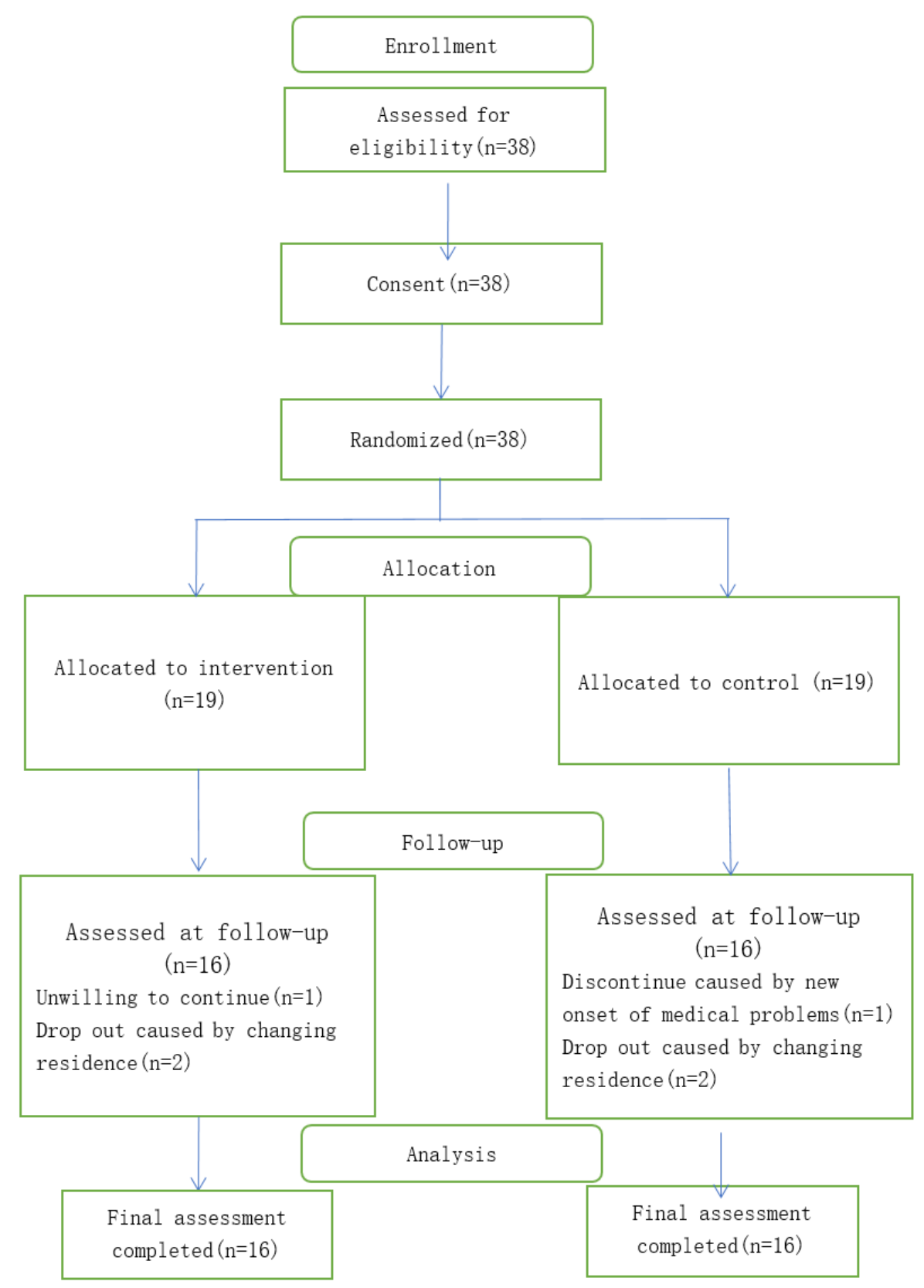

\section{Figure 1}

The flowchart for this study 


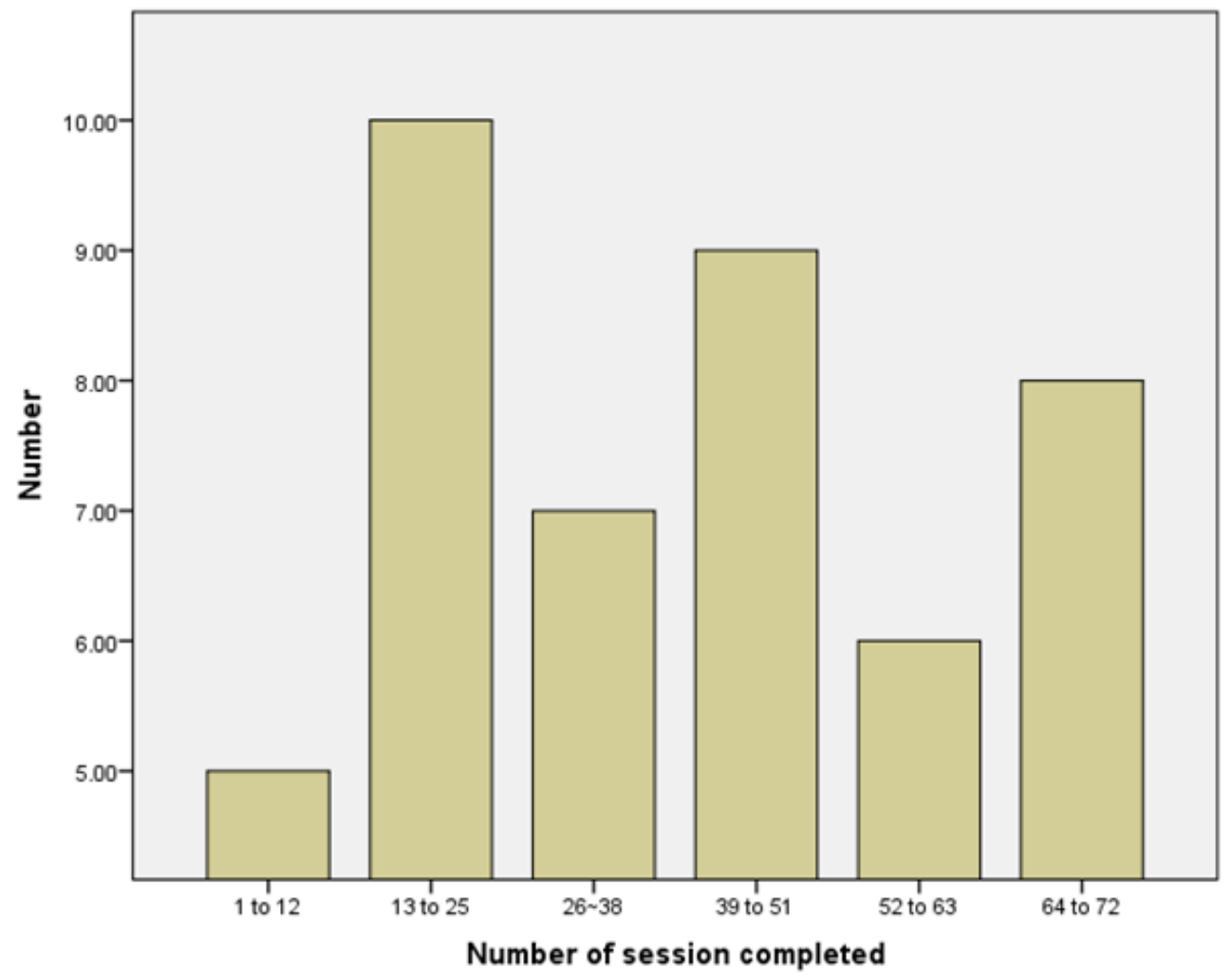

Figure 2

Number of sessions completed by family caregivers-led 